\title{
Signature based Searches for new physics involving photons at the Tevatron
}

\section{R.E. Blair ${ }^{* \dagger}$}

Argonne National Laboratory, Argonne, Illinois

E-mail: rebeanl.gov

\begin{abstract}
We present a variety of model-independent studies of final states involving photons in combination with other objects. These include charged leptons (including taus), jets (including b-tagged jets), additional photons, and missing energy. Several kinematic distributions are examined in each final state considered to search for discrepancies from the standard model. One of the final states examined, involving a photon, a charged lepton, a b-tagged jet, and missing energy, is employed to study standard model production of t-tbar-gamma in addition to potential new physics. The results use data collected at the Tevatron
\end{abstract}

35th International Conference of High Energy Physics

July 22-28, 2010

Paris, France

* Speaker.
${ }^{\dagger}$ For the CDF and D0 Collaborations.




\section{Introduction}

A number of signatures for models that extend the standard model (SM) include final state photons. Processes associated with SUSY, Technicolor and associated Higgs production are all expected to yield events with photons and other associated objects. By comparing the rate of photons plus various combinations of leptons, heavy flavor and missing energy to those expected from SM sources it is possible, in a model independent way, to look for signs of new physics. A number of these surveys have been done at the Tevatron by the CDF collaboration. D $\varnothing$ has not reported recently on such model independent searches so the results described here will be exclusively from the CDF experiment.

\section{Diphotons plus $\mathbf{X}$}

The first search we describe is one reported in [1]. The event selection starts with two triggered photon candidates (two isolated candidates above $12 \mathrm{GeV}$ or two above $18 \mathrm{GeV}$ with no isolation requirement). Events are required to have two photon candidates with $E_{T}>13 \mathrm{GeV}$ and $0.05<$ $|\eta|<1.05$. Both candidates must have a shower maximum lateral profile consistent with a single shower, no high PT tracks pointing at the candidate, isolation (track and calorimeter) in an $\eta, \phi$ cone of $R<0.4$. The calorimeter isolation cut is at $0.1 E_{T}$ for $E_{T}<20 \mathrm{GeV}$ or $2.0 \mathrm{GeV}+0.02\left(E_{T}-\right.$ $20 \mathrm{GeV}$ ) above $20 \mathrm{GeV}$. The track isolation cut is $2.0 \mathrm{GeV}+0.005 E_{T}$. The sample corresponding to $2.0 \pm 0.1 \mathrm{fb}^{-1}$ of running consists of 31,116 events with approximately $30 \%$ of these events having true diphotons.

\subsection{Diphoton plus leptons}

For associated production of leptons and diphotons a $\tau$ candidate was identified by identifying a $\pi^{0}$ candidate in the shower maximum chamber in the electromagnetic calorimeter and combining it with tracks in an energy dependent cone (e.g. $\theta<0.17$ for $E_{T}=30 \mathrm{GeV}, \theta<0.05$ for $E_{T}=$ $100 \mathrm{GeV}$ ). The tracks plus $\pi^{0}$ must have a mass less than $1.8 \mathrm{GeV} / \mathrm{c}^{2}$. In addition the candidate $\tau$ must have less than $1.0 \mathrm{GeV}$ of track $P_{T}$ and less than $0.6 \mathrm{GeV}$ of electromagnetic energy in an ring around the candidate with $R<.52$ in $\eta, \phi$. Thirty four events are observed. The expected number of events is $46 \pm 10$ dominated by fake $\tau$ candidates ( 44 of the 46 expected).

Table 1 lists the expected event rates and the observed rates for diphoton events with an associated electron or muon candidate. These events are from a subsample of the above dataset corresponding to $1.1 \pm 0.1 \mathrm{fb}^{-1}$ with a lepton $E_{T}$ cut of $20 \mathrm{GeV}$.

\subsection{Diphoton plus missing $E_{T}$}

A detailed understanding of jet resolution and underlying event contributions has been used to provide an estimate of the $\mathbb{E}_{T}$ significance. This is defined as $-\log (P)$ where $P$ is the probability of the fake $\mathbb{E}_{T}$ being larger than the observed $\mathbb{E}_{T}$ given the measured event characteristics, jets and unclustered energy. Figure 2 shows the distribution of the significance for the diphoton sample and above a significance of about 4 it can be seen that the dominant contribution to the event rate is 

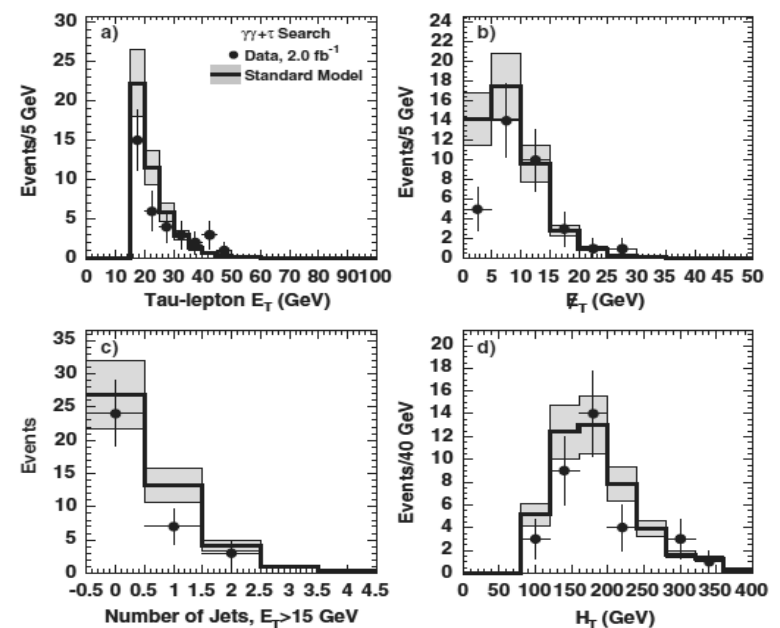

Figure 1: Several distributions from the sample of $\tau$ candidates detected in association with a pair of photons.

\begin{tabular}{|l|l|l|}
\hline Source & electron & muon \\
\hline$Z \gamma \gamma$ & $0.82 \pm 0.08$ & $0.50 \pm 0.05$ \\
\hline$W \gamma \gamma$ & $0.15 \pm 0.02$ & $0.08 \pm 0.01$ \\
\hline$l \gamma+e \rightarrow \gamma$ & $2.26 \pm 0.46$ & $0.004 \pm 0.004$ \\
\hline$l \gamma+$ jet $\rightarrow \gamma$ & $0.44 \pm 0.26$ & $0.12 \pm 0.08$ \\
\hline Fakel $+\gamma \gamma$ & $0.12 \pm 0.05$ & $0.004 \pm 0.004$ \\
\hline Total & $3.79 \pm 0.54$ & $0.71 \pm 0.10$ \\
\hline Observed & 1 & 0 \\
\hline
\end{tabular}

Table 1: A compilation of the primary sources of events in the $e / \mu$ plus diphoton sample.

\begin{tabular}{|l|l|l|l|}
\hline & significance $>\mathbf{3}$ & significance $>\mathbf{4}$ & significance $>\mathbf{5}$ \\
\hline Electroweak & $35.4 \pm 2.2$ & $29.9 \pm 2.0$ & $25.9 \pm 1.9$ \\
\hline Total expected & $71.7 \pm 7.5$ & $39.0 \pm 3.1$ & $30.4 \pm 2.4$ \\
\hline Observed & 82 & 31 & 23 \\
\hline
\end{tabular}

Table 2: Event rates and expected rates in the $\mathbb{E}_{T}$ plus diphoton sample.

from events with real $\mathbb{E}_{T}$. Table 2 shows the expected and observed event rates for three cuts on significance in this sample,

\section{3. $\gamma$ plus jet plus b plus $\mathbb{E}_{T}$}

The analysis in this section is described in [2]. The events are from a $2.0 \mathrm{fb}^{-1}$ sample. Events are required to have a photon candidate with $E_{T}>25 \mathrm{GeV}$ and $|\eta|<1.1$, two jets with $E_{T}>$ $15 \mathrm{GeV}$ and $|\eta|<2.0$, separation of $R>0.4$ for the jets and photon candidate, more than $25 \mathrm{GeV}$ 


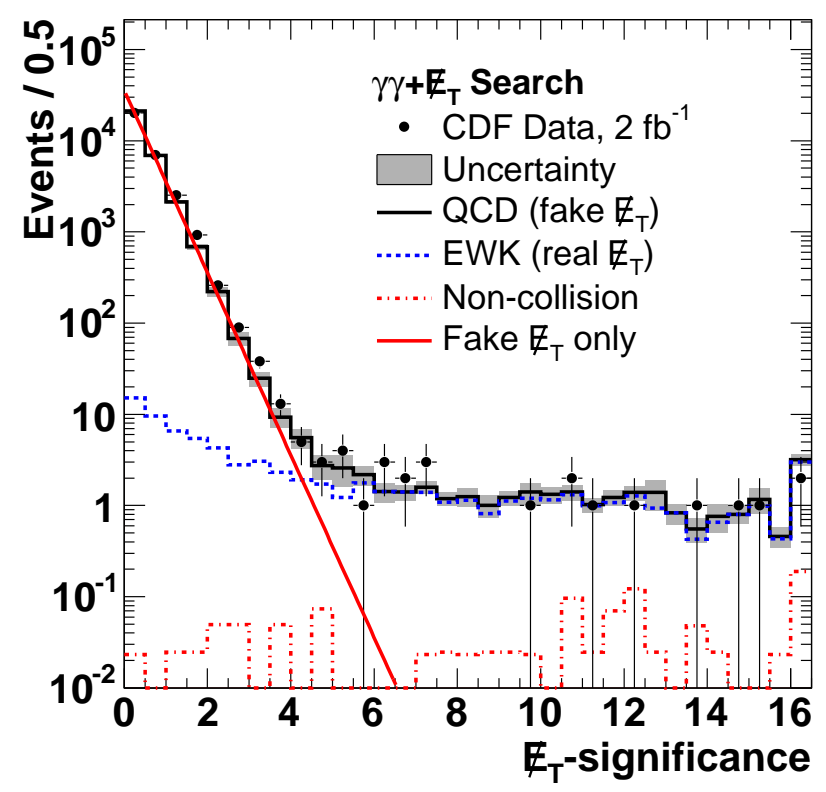

Figure 2: The $\mathbb{E}_{T}$ significance for the diphoton sample along with various various estimates for standard model event contributions to this distribution.
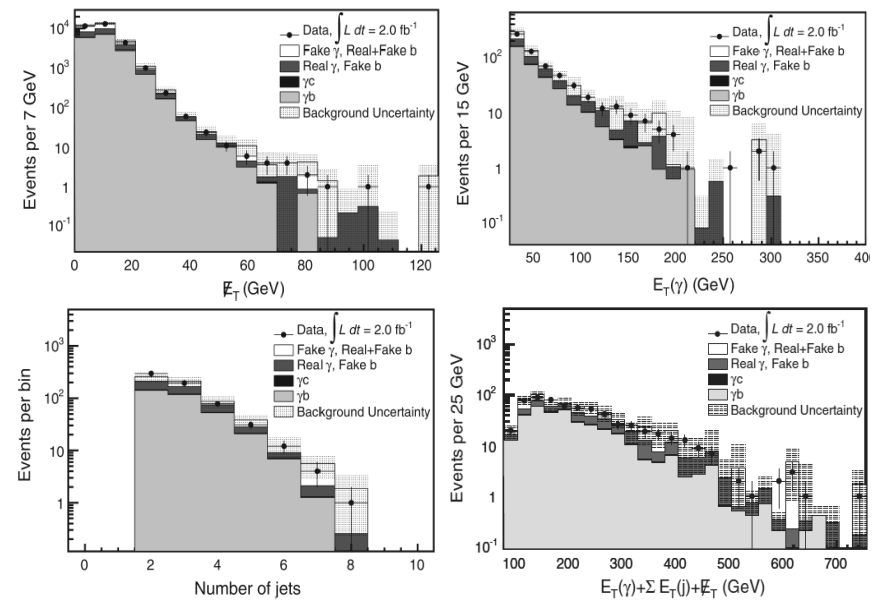

Figure 3: Distributions of the $\mathbb{E}_{T}$, photon $E_{T}$, number of jets and sum $E_{T}$ in the $\gamma+\mathrm{b}+\mathrm{jet}+\mathbb{E}_{T}$ sample.

of $\mathbb{E}_{T}$ with the $\mathbb{E}_{T}$ angle from the jets greater than 0.3 radians in $\phi$. In addition the events had to have a b-tag. the total sample satisfying these cuts was 617 events. The expected number was $607 \pm 74$ (stat.) \pm 86 (syst.) which includes $115 \pm 49 \pm 54$ fake photons and $141 \pm 6 \pm 30$ true photons with fake b-tags. The expected number of true photons plus true b-jets dominates at $341 \pm 18 \pm 91$. Figure 3 shows some of the event sample characteristics. 

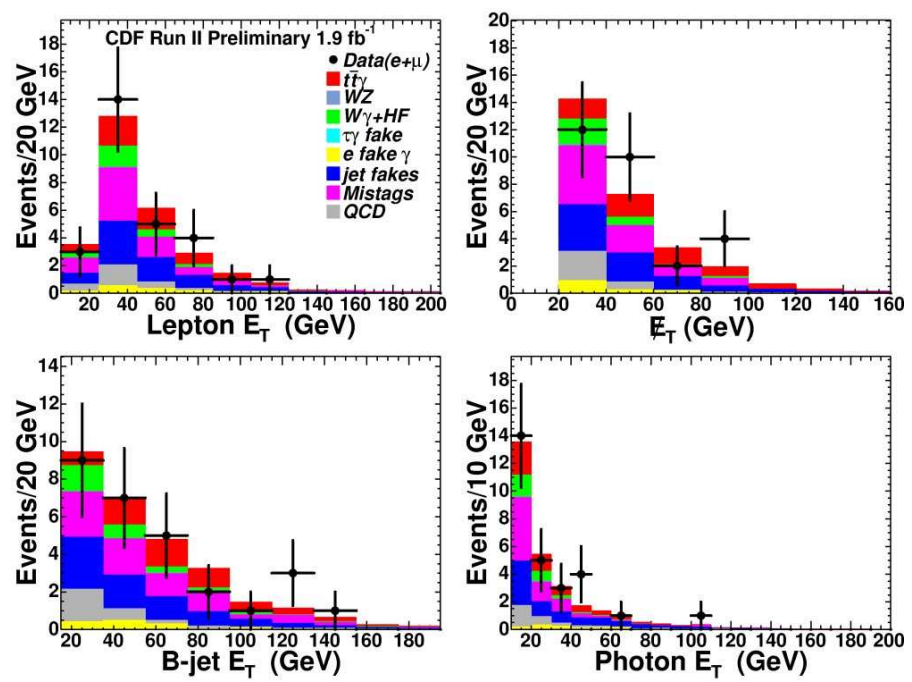

Figure 4: Distributions of the $E_{T}$ of lepton, b jet, $\mathbb{E}_{T}$ and photons in the events with a $\gamma+\mathrm{b}+\mathbb{H}_{T}+$ lepton.

\section{4. $\gamma$ plus b plus $\mathbb{E}_{T}$ plus lepton (e or $\mu$ )}

The final sample described here is discussed in [3]. This corresponds to a $1.9 \mathrm{fb}^{-1}$ sample triggered on high $P_{T}$ leptons. Events were required to have a central photon with $E_{T}>10 \mathrm{GeV}$, a b-tagged jet with $E_{T}>15 \mathrm{GeV}, \mathbb{E}_{T}>20 \mathrm{GeV}$ and an e or $\mu$ candidate with $E_{T}>20 \mathrm{GeV}$. twenty eight events are observed, the expected number of events in this sample is $31.0_{-3.9}^{+4.1}$. The expected event rate is dominated by jets faking photons and mistagged $b$ jets $(7.58 \pm 3,11$ and $7.65 \pm 0.70$ respectively). The next highest contribution is top plus a photon (semileptonic: $3.58 \pm 0.65$ and dilepton: $2.32 \pm 0.41$ ). Figure 4 shows some of the characteristics of the event sample compared to the expected sources.

By cutting on $H_{T}$ in this sample the top fraction of this sample can be enhanced. The number of events after a cut on $H_{T}>200 \mathrm{GeV}$ and 3 or more jets is 16 with 4 expected from top plus a photon and 11.2 $2_{-2.1}^{+2.3}$ expected total. By subtracting the non-top sources an estimate of the top plus gamma cross section can be obtained. That estimate is $0.15 \pm 0.08 p b$ for the $t \bar{t} \gamma$ process.

\section{Conclusions}

A number of searches for unexpected excesses in events with photons plus other signatures was performed at the Tevatron. No significant excesses have been observed. The Tevatron data sample is sufficiently large to show some sensitivity to such extraordinarily rare processes as top plus photon production.

\section{References}

[1] T. Aaltonen et al. [CDF Collaboration], Phys. Rev. D 82, 052005 (2010) [arXiv:0910.5170 [hep-ex]].

[2] T. Aaltonen et al. [CDF Collaboration], Phys. Rev. D 80, 052003 (2009) [arXiv:0905.0231 [hep-ex]].

[3] T. Aaltonen et al. [CDF Collaboration], Phys. Rev. D 80, 011102 (2009) [arXiv:0906.0518 [hep-ex]]. 